\title{
Semi-Automatic Objects Recognition in Urban Areas Based on Fuzzy Logic
}

\author{
Federico Prandi, Raffaella Brumana, Francesco Fassi \\ Department of B.E.S.T., Politecnico di Milano, P.zza Leonardo da Vinci, Milan, Italy \\ E-mail: \{federico.prandi, raffaella.brumana,francesco.fassi\}@polimi.it
}

\begin{abstract}
Three dimensional object extraction and recognition (OER) from geographic data has been definitely one of more important topic in photogrammetry for quite a long time. Today, the capability of rapid generating high-density DSM increases the supply of geographic information but the discrete nature of the measuring makes more difficult to recognize correctly and to extract 3D objects from these surface. The proposed methodology wants to semi-automate some geographic objects clustering operations, in order to perform the recognition process. The clustering is a subjective process; the same set of data items often needs to be partitioned differently based on the application. Fuzzy logic gives the possibility to use in a mathematical process the uncertain information typical of human reasoning. The concept at the base of our proposal is to use the information contained in Image Matching or LiDAR DSM, and typically understood by the human operator, in a fuzzy recognition process able to combine the different input in order to perform the classification. So the object recognition approach proposed in our workflow integrates 3D structural descriptive components of objects, extracted from DSM, into a fuzzy reasoning process in order to exploit more fully all available information, which can contribute to the extraction and recognition process and, to handling the object's vagueness. The recognition algorithm has been tested with to different data set and different objectives. An important issue is to apply the typical human process which allows to recognize objects in a range image in a fuzzy reasoning process. The investigations presented here have given a first demonstration of the capability of this approach.
\end{abstract}

Keywords: Objects Recognition, DSM, Fuzzy Logic

\section{Introduction}

\subsection{Motivations}

Estimating the research fields involved in the 3D topographic objects generation for 3D GIS are very huge.

3D GIS require 3D representations of objects and 3D object reconstruction of real world is a relatively new issue in photogrammetry.

Progress in the field of digital image matching for DSM generation and growth of airborne laser scanning technologies have improved the derivation of digital surface models.

The basic idea of this work is to use the 3D information contained in these DSM in order to aid the 3D identification and extraction of features. Using the DSM we would to overcome some typical difficulties in image interpretation.

Then this work focuses on the automation and accel- eration of the recognition of 3D topographic objects for 3D GIS. The purpose is to use the 3D information contained in the DSM in order to automatic detect and recognize topographic objects in complex scenes. Furthermore, it is tried to use only LiDAR or Image Matching DSM. Due to this limitation our goal becomes even more challenging.

Experience and daily practice allow us to have our brain automatically interpreting what we see. Human recognition takes advantage of a variety of acquired information rather than relying on, say, a single descriptor of the objects. Further, human perception possesses a tremendous potential for learning and deals perfectly with the fuzziness of the real world. In the case of objects in geographic data an interpretation by human operator may be easier but, if these processes are replaced by a computer, it is more than probable that none of the objects will be identified.

Fuzzy set theory, introduced by L. Zadeh in the 1960s, resembles human reasoning in its use of approximate 
information and uncertainty to generate decisions.

This work contains possible methods that aim at an automated object recognition based on fuzzy logic. An important aspect of fuzzy reasoning is that the rule base should include observations of the important object's descriptors. Moreover, it reflects the fact that people may formulate similar "fuzzy statements" to characterize how they perceive object in range images. Formulating the rules is more a question of the expertise of an operator than of a detailed technical modelling approach. This aspect is very important because we are aware of the fact that an operator will never be completely replaced.

\subsection{Related Works}

Three dimensional object extraction and recognition (O ER) from geographic data has been definitely one of more important topic in photogrammetry for quite a long time. However, most of the existing methods for automatic object extraction and recognition from data are just based on the range information and employ parametric methods while object's vagueness behaviour is basically neglected [1].

The main task in automatic DTM generation from data is the separation of terrain points from non-terrain points; further classification of non-terrain points in buildings, vegetation and other objects (e.g. bridges) may be necessary, depending on the application.

Since long years automatic approaches to DTM generation and building reconstruction based on radiometric images have long been a research topic. It is shown that the segmentation and classification problem is not only an interesting research topic, but also very important in practice. Multiple, largely complementary, sensor data such as colour or multi-spectral aerial images and range data from laser scanners or SAR have been used to achieve robustness and better performance in 3D ORR. For example, colour infrared (CIR) aerial images in combination with laser scanner data DTMs can be used for feature extraction [2].

If multispectral imagery is available the classification approach is the most convenient way for detecting building areas or urban regions [3]. Everything that exceeds a certain threshold in the nDSM will be included, and vegetation will be afterwards excluded by interpreting the NDVI [4].

In many case however the multispectral information are absent and techniques in order to segment the DSM are carried out. Different filtering strategies have been proposed, based on deviations from a parametric surface, slope threshold, clustering, etc.

A slope based filtering using mathematical morphology has been developed defining a slope threshold as the maximum allowed height difference between two points as a function of their spatial distance [5].
A complex iterative procedure, based on the analysis of gradient, surface orientation and residuals from successive spline interpolations has been implemented in order filtered out non-terrain points [6].

Another interesting approach [7] has been discussed: the segmentation is carried out by combining region growing with a principal component analysis (PCA).

A three-stage framework has been implemented for a complete, robust and automatic classification of LiDAR data and is composed by: a region-growing technique to obtain regions with a step edge along their border, a grouping of connected sets of pixels on the basis of an 8-classes partition of the height gradient orientation and a rule based scheme applied to the classification of the regions [8].

Study focuses on the automatic extraction of a DTM from a high-resolution DEM produced by image correlation in urban or rural areas based on a hybrid approach has been designed and combines complementary aspects of both TIN-based and segmentation-based techniques [9].

This review outlines the considerable efforts which have been undertaken to deal with the complex problems of segmentation Computational theories on human perception and artificial intelligence are partially included in these developments.

\section{Classification Based on Fuzzy Logic}

\subsection{Fuzzy Logic and Application in Feature Extraction}

By recognition we refer to the process that assigns a label (e.g. "building” or "tree”) to a segmentation result, in particular a region, based on properties (descriptors) of the region. Segmentation is the process that partitions the spatial domain of an image or other raster datasets like digital elevation models into mutually exclusive parts, called regions.

The major challenge of this work is to develop a segmentation procedure in connection with an inference process for object recognition where computational theories on human perception and artificial intelligence are partially included.

The bridge to connect the computational theories specified above with the human perception can be given by the Fuzzy Logic.

Fuzzy logic provides a simple way to arrive at a definite conclusion based upon imprecise, uncertain, ambiguous, vague or missing input information. Over the past few decades, fuzzy logic has been used in a wide range of problem domains. Although the fuzzy logic is relatively young theory, the areas of applications are very wide: process control, management and decision making, operations research, economies. Dealing with simple 'black' 
and 'white' answers is no longer satisfactory enough; a degree of membership (suggested by Prof. Zadeh in 1965) became a new way of solving the problems. The natural description of problems, in linguistic terms, rather than in terms of relationships between precise numerical values, is the another advantage of this theory.

Fuzzy Logic introduces linguistic variables for the object characteristic descriptors and linguistic labels to describe the fuzzy sets on the range of all possible values of the linguistic variables.

The basic idea of the recognition approach presented in this work is to resemble human reasoning in its use of imprecise, vague or missing information. This involves formulating rules with imprecise knowledge and providing a simple way of arriving at a definite conclusion, i.e. the recognized objects. The recognition approach based on fuzzy reasoning and a concept for introducing learning into fuzzy recognition is in the focus of this work.

\subsection{Application in Feature Extraction}

Many methods in pattern recognition and feature extraction have been proposed, based on various approaches, such as Bayesian inference, neural networks, linear methods, nearest prototypes, etc. Fuzzy set theory and related domains have brought new tools for pattern recognition, either based on the concept of fuzzy sets as a fundamental concept for modelling classes, or based on non classical representations of uncertainty.

These approaches can often be viewed as a generalization of some classical method, these generalizations introducing either some fuzziness in the modelling or some uncertainty measure more general than probability measures. In other cases, they follow the same kind of philosophy, but with different tools. Typical examples in the first category are: the fuzzy $k$-nearest neighbours and the fuzzy $c$-means. In the second one, the fuzzy pattern matching approach, in the framework of possibility theory, can be viewed as the possible counterpart of the Bayesian approach to classification.

Applied to raster image, fuzzy classification estimates the contribution of each class in the pixel. It assumes that a pixel is not an indecomposable unit in the image analysis and, consequently, works on a new principle: "one pixel-several classes" to provide more information about the pixel unlike the hard classification methods which are poor in information extraction. For instance a fuzzy c-means clustering algorithm has been developed and implemented in unsupervised classification of remote sensing data [10].

In the proposed fuzzy process (Figure 1), each pixel is transformed into a matrix of membership degrees representing the fuzzy inputs. A minimum-reasoning rule is, then, applied to infer the fuzzy outputs. Finally, a defuzzification step is applied to extract features [11].
The main components of the fuzzy recognition process are as follows:

- a database, which defines the membership functions of the fuzzy sets,

- a rule base, which contains fuzzy if-then rules,

- a fuzzy reasoning procedure, which performs inference operations on the rules.

This should consider 1) all available descriptors of an object (such as: 3D structure, textural information and spectral responses), 2) a fuzzy description of object properties and a fuzzy inference strategy for object recognition, 3) learning capabilities to modify imprecise model descriptions and increase the potential of recognition in particular if new and unrecognized objects are encountered.

The fuzzy AND or OR operators combine the membership values of the inputs in each rule for the antecedent of that rule. The MIN reasoning rule, applied on the matrix of produced fuzzy, inputs will consider, for each class, the membership degrees provided by the different fuzzy sets and pick out the minimal membership degree to represent the class extent in the pixel. Then a MAX operation will be performed, on the consequent of each rule, to obtain the element with the highest value (fuzzy output) and the corresponding class of that feature willbe considered as associated fuzzy class to that pixel (Figure 2).

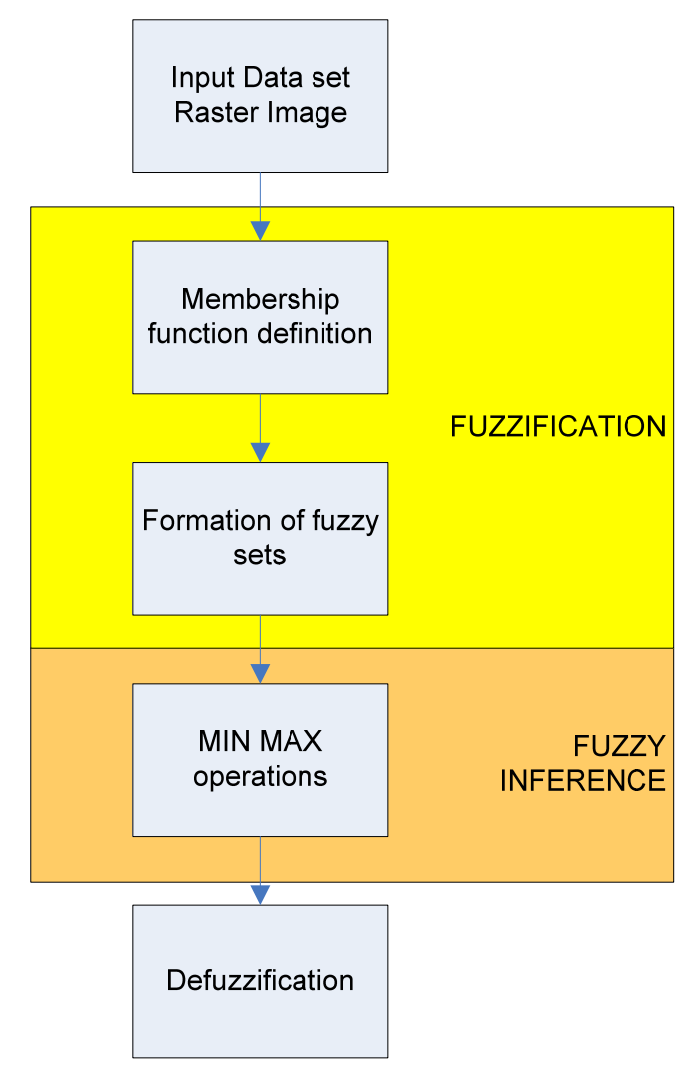

Figure 1. Implementation flowchart in fuzzy step. 
Rule base should include observations of the important descriptors. Moreover, it reflects the fact that people may formulate similar "fuzzy statements" to characterize how they perceive objects in, for instance, aerial colour images.

The investigations of the works involved in classification and feature extraction [12-14] have given a demonstration of the capability of these fuzzy approaches.

\subsection{Proposed Workflow}

The proposed methodology wants to semi-automate some geographic objects clustering operations, in order to perform the recognition process.

The clustering is a subjective process; the same set of data items often needs to be partitioned differently based on the application. There is no general algorithm or approach that solves every clustering problem. In order to overcome this problem is important to have simple and semi-automated tools with the possibility to change the classification based on different situations and different data.

As seen in the previous, fuzzy logic gives the possibility to use in a mathematical process the uncertain information typical of human reasoning. The concept at the base of our proposal is to use the information contained in Image Matching or LiDAR DSM, and typically understood by the human operator, in a fuzzy recognition process able to combine the different input in order to perform the classification.

So the object recognition approach proposed in our workflow integrates 3D structural descriptive components of objects, extracted from DSM, into a fuzzy reasoning process in order to exploit more fully all available information, which can contribute to the extraction and recognition process and, to handling the object's vagueness.

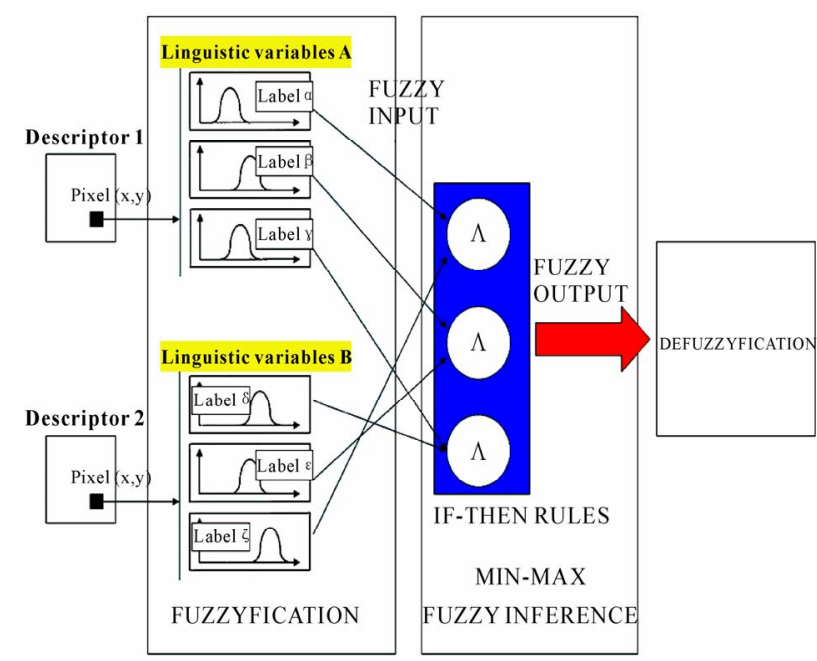

Figure 2. Architecture of the explicit fuzzy method illustrated for two descriptor, two variables with three classes.
The proposed objects extraction method requires some preliminary step that consists in 1) to locate and separate all 3D objects from the terrain and 2) to considerate and generate all the geometric properties that can describe the objects. Then the challenge is to develop a segmentation procedure in connection with an inference process for object recognition.

Basic idea was to perform the classification procedure using a priori human knowledge about the objects and then in fuzzy logic manner. Some information, needed for membership function definition, was taken from human heuristic knowledge.

More specifically the steps of fuzzy recognition process will be:

- input raster data and structural descriptor variables are introduced;

- membership functions are defined using results from human heuristic knowledge;

- definition of fuzzy logic inference rules;

- performing Raster data classification.

The OER process starts with the extraction of the Structural Descriptor (SD). Then the descriptors are analyzed to derive the SD membership functions. In the next stage, the recognition operation is performed with the application of the sample if-then fuzzy rules and the MIN-MAX inference process (Figure 3).

Prior to fuzzy recognition the membership functions of the structural descriptors have to be specified but, before the system operation is started, it is always necessary to set up the fuzzy reasoning parameters. For the object classes, the preliminary Structural descriptor's membership functions components are defined based on the knowledge of an experienced photogrammetric operator.

The formulation of the fuzzy rules requires a profound observation of the integrative impact of the descriptors on the recognition of an object. This again depends on the experience of an operator but also on the complexity of an object. In the experiments we observed that for partition walls a comparatively small number of fuzzy rules are sufficient, whereas trees require more rules probably due to their more complex shapes and variety of appearances.

\subsection{Recognition of Little Objects in Urban Areas: The Partition Walls}

In the data-test we investigated the possibility to recognize large scale objects in urban areas. In these areas large amount of objects are constituted by building and road feature classes but, there are also many little objects which are important i.e. for construction of 3D city models. In many cases these features are difficult to single out into images by an expert photogrammetric operator too. The partition wall is a typical example of this kind of feature class. In fact due to their geometry is difficult 


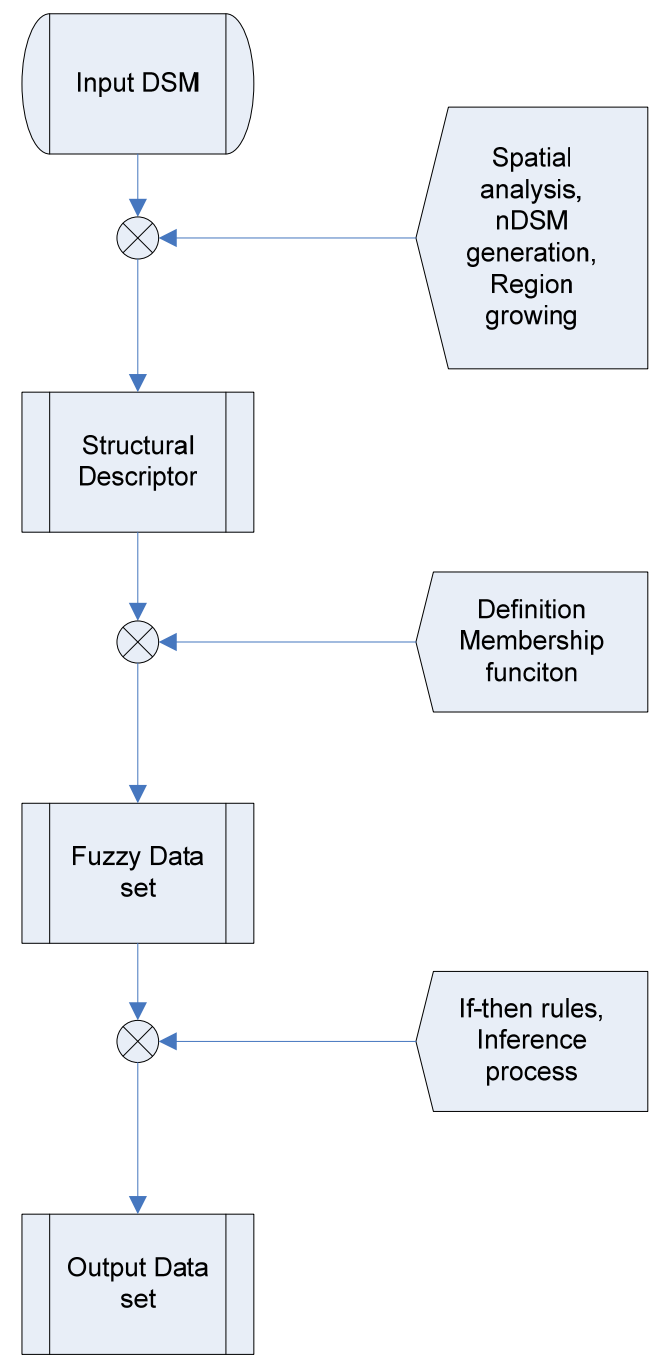

Figure 3. OER (Object Extraction and Recognition) fuzzy process work-flow.

to detect them into image, beside they may have parts occluded by the buildings or trees, or may be confused by the shadow of the walls themselves. Human recognition takes advantage of a variety of acquired information and the walls are identifiable on the aerial images more like as a separation between to different areas then like as single geometry. In this situation human perception possesses a tremendous potential for learning and deals perfectly with the fuzziness of the real world. For these reasons the recognition of these particular features may take advantage from a fuzzy logic approach, which is able to capture the "fuzziness" or "imprecision" of the real world.

Beside in this particular context use of Structural descriptor are fundamental because the spectral information is very poor due to shadows, noise and similarity to other objects such as road, buildings etc. So this is a very important challenge for our approach which starts only from 3D Structural Descriptors.
The data test is an urban area of Milan, there are many partition walls which divide road and railways and like as boundary of big factory (Figure 4). The input DSM is obtained trough Image matching technique and has $1 \times 1$ meter resolution.

The surface model generated by image matching is shown in (Figure 5). Appling segmentation of the surface model based on morphological filtering in order to the detected 3D regions, we can obtain the nDSM.

After refinement of the extracted regions the next step is to derive the structural descriptors: gradient edge detector and others. The membership functions are defined and applied to these features and the results are shown in Figure 6.

The trapezoidal membership function, with maximum equal to 1 and minimum equal to 0 , is used in this work. Special cases like symmetrical trapezoids and triangles reduce the number of parameters to three. The trapezoidal function are modelled with four parameters $(\alpha, \beta, \gamma, \delta)$.

$$
\mu_{A}(x, \alpha, \beta, \gamma, \delta)=\left\{\begin{array}{cc}
0 & \text { if } x<\alpha \\
\frac{x-\alpha}{\beta-\alpha} & \text { if } \alpha \leq x<\beta \\
1 & \text { if } \beta \leq x<\gamma \\
\frac{\delta-x}{\delta-\gamma} & \text { if } \gamma \leq x<\delta \\
0 & \text { if } x<\delta
\end{array}\right.
$$

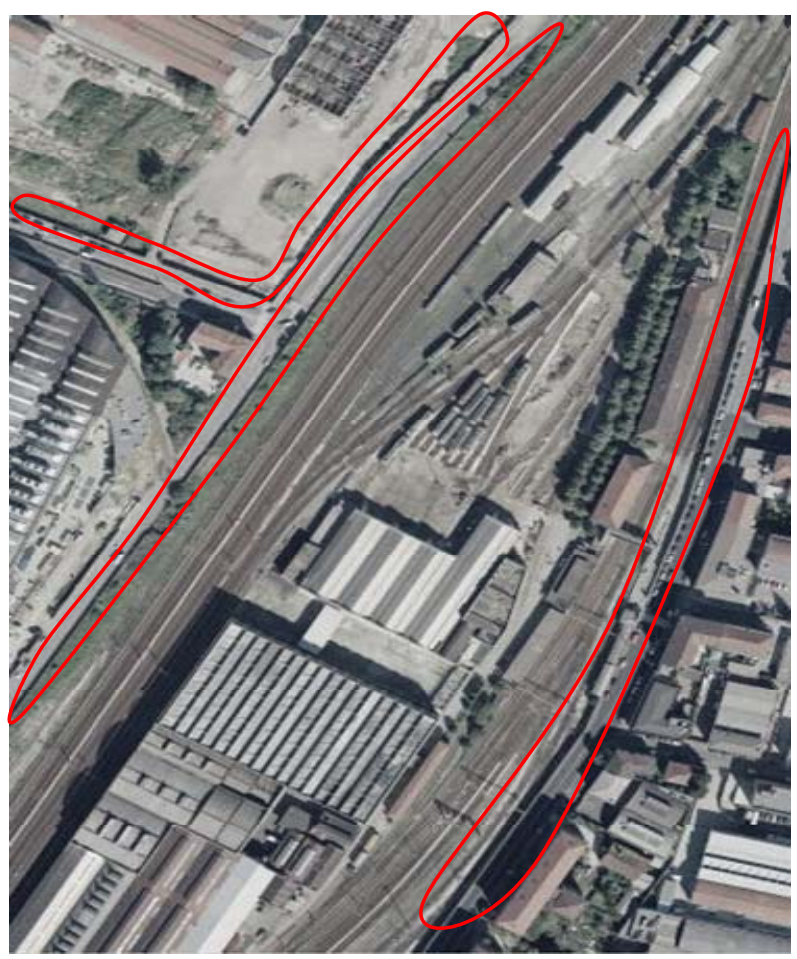

Figure 4. Sample data-set, the more important partition walls are highlighted. It is possible to see like as the object identification into the image is problematic. 


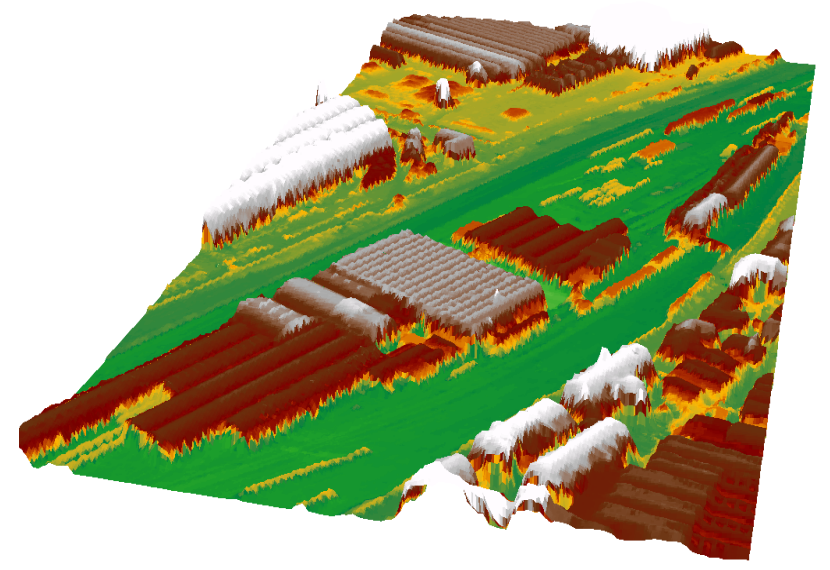

Figure 5. Digital surface model generated by image matching. $1 \times 1$ meter resolution.
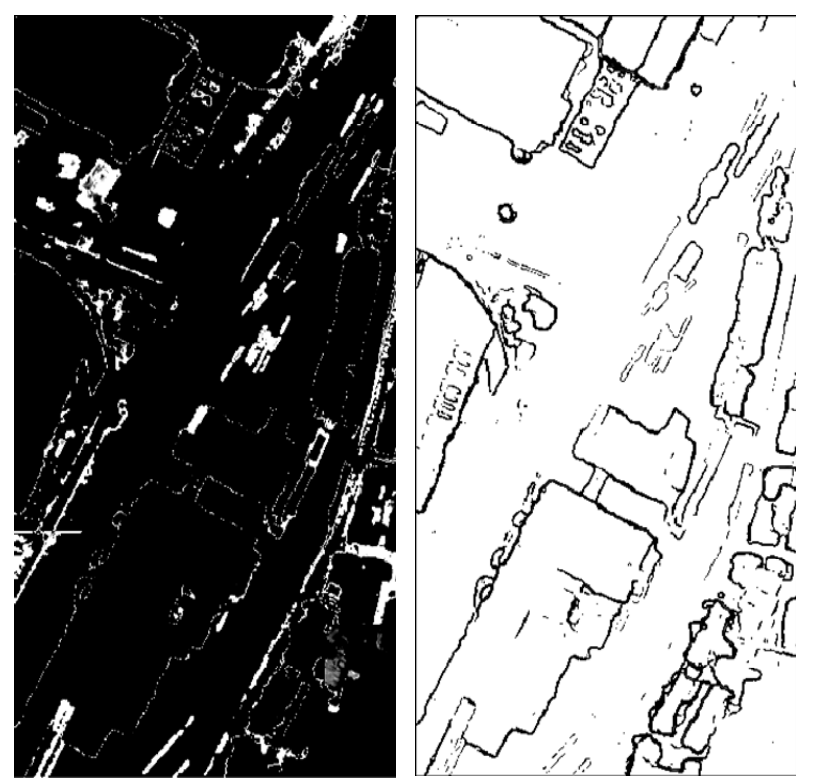

Figure 6. Application of the membership function to the structural descriptors (Height range, edge) generation of the fuzzy Data Set.

The linguistic variables, which are variables that assume linguistic terms values, of the object's structural descriptors have to be defined in fuzzy logic. Linguistic variables are associated to each input structural descriptor and for each linguistic variables some linguistic labels has been assigned. This assignment is mostly a mixture of expert knowledge and examination of the desired inputoutput data.

Through definition of linguistic variables and membership function parameters calculation of the input to the fuzzy recognition process is given.

In a second step the determined membership values have to be combined to get a final decision (inference process). This component of the fuzzy recognition process consists in the definition of a set of rule base, which contains fuzzy if-then rules.

Once defined the sample if-then rules we can start the inference process. The results of classification are shown in Figure 7.

This methodology returns as output a very thigh recognition objects set, due to use of min implication, which consist in low pass filter. In this situation is enough a wrong value in an input data set to get worse in the objects extraction. For instance, inaccurate DTM extraction may cause the elimination of some objects part from nDSM. This is all the more probable in case of little objects such as walls. The result is a chopped classification set where the objects classified as walls are correctly understood and the false positive are limited but, we have many not recognized objects and the geometry results fragmented.

The second method we have tested a weighted sum approach [15] and this has shown better results.

The resulting output value (Figure 8 left) is calculated by the sum of the membership values for each rules concerning an individual weight factor $W_{i}$. The weights for parameters height, edge and height range depend on the experience and data input/output

$$
\begin{aligned}
& \qquad m_{i}=\mu_{A_{i}}\left(x_{1}\right) \cdot W_{A_{i}}+\mu_{B_{i}}\left(x_{2}\right) \cdot W_{B_{i}}+, \ldots \ldots .,+\mu_{N_{i}}\left(x_{n}\right) \cdot W_{N_{i}} \\
& \text { with } \sum_{i=1}^{n} W_{i}=1
\end{aligned}
$$

Our investigations have shown that the classified objects are better defined than in fuzzy-min approach and we may recognize more features than previously.

Finally an accuracy investigation is carried out to evaluate the recognition results. For this purpose the ex-

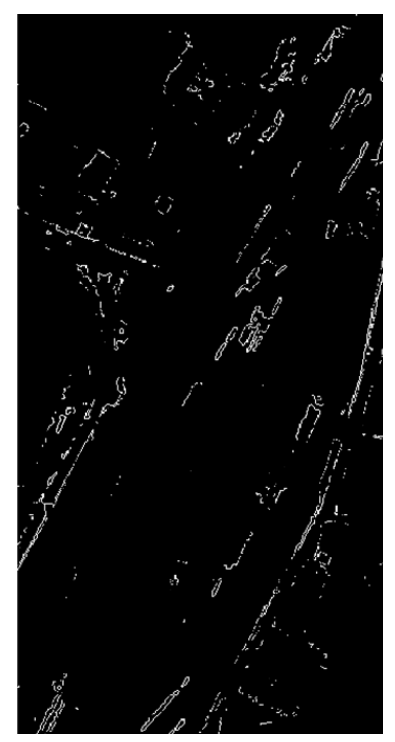

Figure 7. Results of fuzzy walls recognition process. 

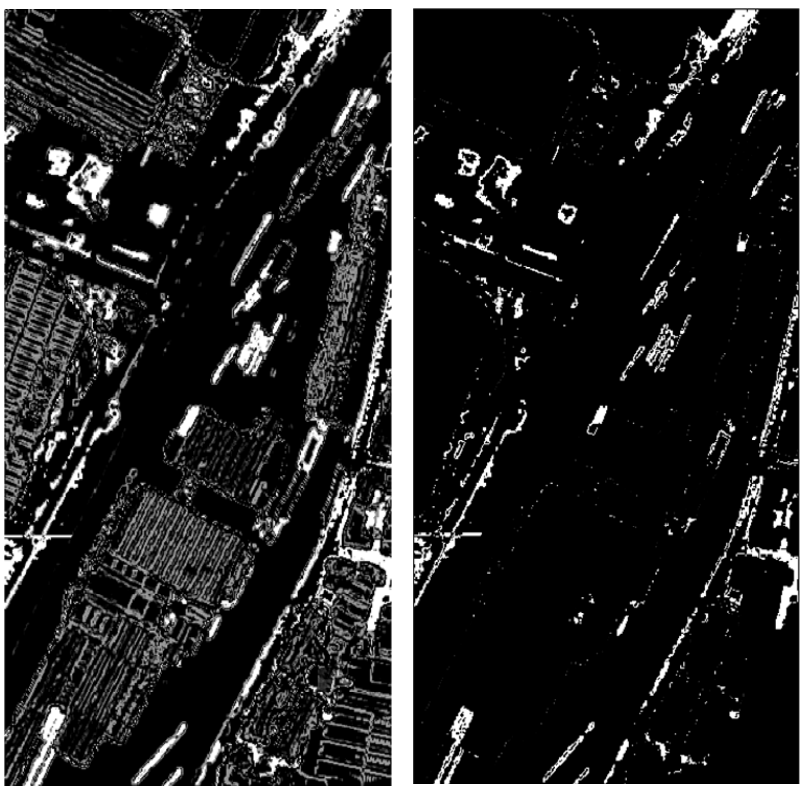

Figure 8. Results of fuzzy walls recognition process using the weighted sum approach. At left is shown the results of fuzzy inference process and at right the final cluster once applied a threshold (value 0.6 ) to the weighted fuzzy output.

tracted objects are compared to the object manually measured and contained in the 1:1000 digital maps of Milan.

Due to the particular wall's geometry (very thigh in one direction), the classification is influenced by the quality of input DSM. Where DSM is well defined (Figure 9) we have a good result.

\section{Conclusions and Future Works}

In object recognition, human interaction remains an important part of the work flow even though the amount of work to be done by the human operator can be reduced considerably in the global extraction process. Many of automated and semi-automated methods proposed in literature focus their efforts on the reconstruction process or on the feature extraction once recognized the objects by the operator.

The core of the system is an approach for recognition of object's primitives based on fuzzy logic theory which analyzes the data in order to extract a maximal amount of information, through an explicit process that uses structural information of objects and integrates them in a fuzzy reasoning process.

The methods presented in this work integrate some typical heuristic human concept and is adaptive to operate with different data sets and in different applications. Despite that further investigation and developments might be carried out. In relation to data input different descriptor

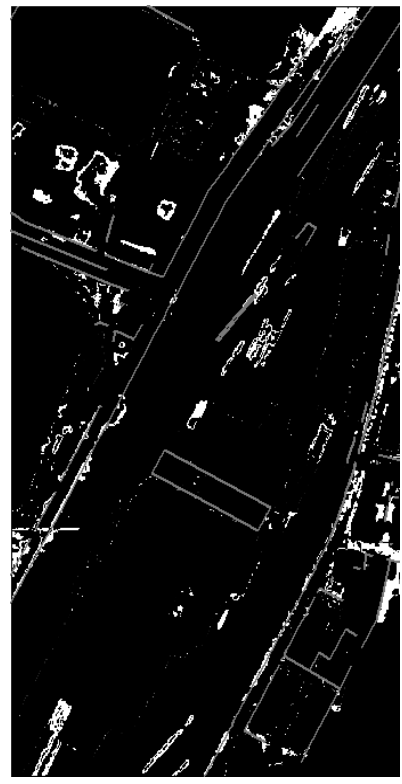

Figure 9. Comparison between the extracted features and 1:1000 digital maps of Milan. In red the wall features manually extracted. We can observe a good result for $\mathbf{5 0 \%}$ of elements, while some walls are not recognized and we have many false-positive due to the presence of many objects which have wall's similar characteristics.

of objects, such as textural or multispectral properties, can be considered in recognition process. Moreover the generation of structural descriptor have to be improved considering the sensitivity of the algorithms to the coarse input.

Another important improvement is neural-network learning. The learning capability of neural networks can be introduced to the fuzzy recognition process by taking adaptable parameter sets into account which leads to the neuro-fuzzy approach.

Finally data acquired from different sensor (such as High density Airborne Laser Scanning) or new systems (UAV) can be used in recognition process and their potentiality can be evaluated.

\section{References}

[1] F. Samadzadegan, A. Azizi, M. T. Hahn and C. Lucas, "Automatic 3D Object Recognition and Reconstruction Based on Neuro-Fuzzy Modelling," ISPRS Journal of Photogrammetry \& Remote Sensing, Vol. 59, No. 5, 2005, pp. 255-277.

[2] M. Hahn and C. Stätter, "A Scene Labeling Strategy for Terrain Feature Extraction Using Multisource Data," International Archives of Photogrammetry and Remote Sensing, Vol. 32, Part 3/1, 1998, pp. 435-443.

[3] T. Knudsen and B. Olsen, “Automated Change Detection for Updates of Digital Map Databases,” Photogrammetric 
Engineering \& Remote Sensing, Vol. 69, No. 11, 2003, pp. 1289-1296.

[4] G. Sohn and I. J. Dowman, "Terrain Surface Reconstruction by the Use of Tetrahedron Model with the MDL Criterion, International Archives of Photogrammetry," Remote Sensing and Spatial Information Sciences, Vol. 34, Part 3A, 2002, pp. 336-344.

[5] G. Vosselmann, "Slope Based Filtering of Laser Altimetry Data," International Archives of Photogrammetry and Remote Sensing, Amsterdam, Vol. 33, No. B3, 2000, pp. 935-942.

[6] M. A. Brovelli, M. Cannata and U. M. Longoni, "Managing and Processing LIDAR Data within GRASS," Proceedings of the Open Source GIS-GRASS Users Conference, Trento, September 2002.

[7] M. Roggero, "Object Segmentation with Region Growing and Principal Component Analysis,” International Archives of Photogrammetry and Remote Sensing and Spatial Information Sciences, Vol. 34, Part 3A, 2002, pp. 289-294.

[8] G. Forlani, C. Nardinocchi, M. Scaioni and P. Zingaretti, "Complete Classification of Raw LIDAR Data and 3D Reconstruction of Buildings," Pattern Analysis Application, Vol. 8, No. 4, 2006, pp. 357-374.

[9] C. Baillard, "A Hybrid Method for Deriving DTMs from Urban DEMs," International Archives of Photogrammetry and Remote Sensing, Vol. 37, Part B3b, 2008, pp. 109-113.

[10] R. L. Cannon, J. V. Dave, J. C. Bezdek and M. M. Trivedi, "Segmentation of a Thematic Mapper Image
Using the Fuzzy C-Means Clustering Algorithm,” IEEE Transactions on Geoscience and Remote Sensing, Vol. 24, No. 3, 1986, pp. 400-408.

[11] F. Melgani, B. A. R. Al Hashemy and S. M. R. Taha, “An Explicit Fuzzy Supervised Classification Method for Multispectral Remote Sensing Images," IEEE Transactions on Geoscience and Remote Sensing, Vol. 38, No. 1, January 2000, pp. 287-295.

[12] J. Amini, C. Lucas, M. Saradjian, A. Azizi and S. Sadeghian, "Fuzzy Logic System for Road Identification Using Ikonos Images,” Photogrammetric Record, Vol. 17, No. 99, 2002, pp. 493-503.

[13] A. Mohammadzadeh, A. Tavakoli and M. J. V. Zoej, "Automatic Linear Feature Extraction of Iranian Roads from High Resolution Multi-Spectral Satellite Imagery," International Archives of the Photogrammetry, Remote Sensing and Spatial Information Sciences, Vol. 35, Part B3, 2004, p. 764.

[14] B. Wuest and Y. Zhang, "Region Based Segmentation Of Quickbird Imagery through Fuzzy Integration,” International Archives of the Photogrammetry, Remote Sensing and Spatial Information Sciences, Vol. 35, Part B7, 2008, p. 491.

[15] T. Vögtle and E. Steinle, “On the Quality of Object Classification and Automated Building Modelling Based on Laser-Scanning Data," International Archives of Photogrammetry Remote Sensing and photogrammetry, Vol. 32, No. 4, 2003, pp. 77-84. 